\title{
Reappraisal of Gestational Weight Gain Recommendations in Obese Pregnant Women: A Population-Based Study of 337,590 Births
}

\author{
Roland Devlieger ${ }^{a}, b, c \quad$ Lieveke Ameye $^{a}$ d Tinne Nuyts $^{a}$ \\ Régine Goemaes ${ }^{e} \quad$ Annick Bogaerts ${ }^{a, f}$ \\ a Department of Development and Regeneration, KU Leuven, Leuven, Belgium; \\ ${ }^{b}$ Department of Obstetrics and Gynecology, University Hospitals Leuven, Leuven, Belgium; \\ 'Department of Obstetrics, Gynecology and Fertility, Gasthuiszusters Antwerpen, Campus \\ St. Augustinus, Antwerp, Belgium; d Data Centre - Biostatistical Unit, Jules Bordet Institute, \\ Université Libre de Bruxelles, Brussels, Belgium; 'e Study Centre for Perinatal Epidemiology \\ (SPE), Brussels, Belgium; ${ }^{f}$ Faculty of Medicine and Health Sciences, Centre for Research and \\ Innovation in Care (CRIC), University of Antwerp, Antwerp, Belgium
}

\section{Keywords}

Gestational weight gain · Recommendations · Obesity classes

\begin{abstract}
Background and Objective: Maternal obesity is an epidemic health problem that is aggravated by excessive gestational weight gain (GWG) and postpartum weight retention. Current US Institute of Medicine (now US National Academy of Medicine) guidelines (2009) for GWG need to be evaluated against the current rise in obesity in the general and pregnant population. We wanted to study the relation between GWG and pregnancy and birth outcomes and to relate this to the current recommendations for GWG. Methods: Population-based study. We performed an epidemiological analysis in a cohort of Belgian pregnant women with singleton live births at term ( $\geq 37$ weeks) between 2009 and $2014(n=337,590)$. Logistic regression was used to determine the optimal GWG in relation to relevant pregnancy and birth outcomes. Results: The prevalence of maternal obesity significantly increased from $10.3 \%$ in 2009 to $11.4 \%$ in 2014 . The mean (SD) body mass index at the start of the pregnancy significantly increased from 23.9 (4.5) in 2009 to 24.2 (4.6) in 2014. Excessive GWG was frequent, especially in overweight (56.8\%) and obese (52.9\%) pregnant women. In the logistic regression mod$\mathrm{el}$, the amount of GWG associated with the lowest incidence of both large-for-gestational-age and small-for-gestational-age infants was $21 \mathrm{~kg}$ in underweight women, $14 \mathrm{~kg}$ in normal weight, $8 \mathrm{~kg}$ in overweight, $0 \mathrm{~kg}$ in obese class I, $-4 \mathrm{~kg}$ in obese class II and $-5 \mathrm{~kg}$ in obese
\end{abstract}


Devlieger et al.: Gestational Weight Gain Recommendations in Obese Pregnant Women

class III. Conclusion: The prevalence of maternal obesity has risen in Belgium between 2009 and 2014. Current GWG guidelines, based on historic observational data, are probably too liberal for class II and III obese women in which better outcomes are being predicted for lower weight gain than recommended.

(C) 2020 The Author(s)

Published by S. Karger AG, Basel

\section{Introduction}

Paralleling the global epidemic of obesity figures in the general population, the incidence of maternal obesity (body mass index, BMI, $>30$ at the start of pregnancy) has been rising in recent decades [1]. While most European countries do not systematically report obesity figures in their pregnant population, the prevalence of maternal obesity varies from 7 to $25 \%$ and seems strongly correlated with social and educational inequalities [2, 3].

Obesity during pregnancy represents an important preventable risk factor for adverse pregnancy outcomes and has a negative impact on long-term health for both mothers and their offspring [4-7]. These effects are often aggravated by a high incidence of abnormal glucose tolerance and excessive gestational weight gain (GWG) in this group. Guidelines for GWG have been developed by the US National Academy of Medicine (NAM) and were revised in 2009 [8]. These guidelines were largely based on epidemiological data gathered 2 decades ago and do not account for the grades of obesity.

Few studies have reported on the evolution of maternal obesity and its consequences in recent years [9]. In the USA, 31.8\% of women aged 20-39 years were obese in 2011-2012 [10]. Rates of overall obesity, in particular class III obesity, have increased significantly between 2005 and 2014 [1]. Unfortunately, nationwide statistics on maternal obesity are not available. Recent studies of American children and adolescents report obesity in $17.0 \%$, with $5.8 \%$ being extremely obese. Since the 1980 s, obesity has been increasing in adolescents; however, in prepubescent children rates appear to be falling [11].

European figures for obesity have traditionally been lower [3]. In Belgium, nearly 1 in 2 adults are overweight, and $14 \%$ are obese. The overall prevalence of female obesity is $14.0 \%$, although this appears to be age specific: $3.5 \%$ (18-24 years), $14.5 \%$ (25-34 years) and 12.5\% (35-44 years). Between 1997 and 2013, adult obesity has increased by 3\%, with the mean BMI increasing from 24.7 to 25.4 . In children (2-17 years), 20\% are overweight, a $5 \%$ increase since 1997 . The highest prevalence for obesity (11\%) is in children aged $2-4$ years [12].

In Flanders, the most northern region of Belgium, perinatal data have been systematically recorded for all deliveries since 1989 . Since 2009, pregestational weight and height as well as weight at delivery were added to the recorded items. Hence, in 2009 the prevalence of women with overweight and obesity was 21.2 and $10 \%$, respectively [2]. We showed an increase in the prevalence of overweight and obese women from 25.4 to $31.4 \%$ between 2 consecutive pregnancies, and in the group of women with obesity at the onset of the first pregnancy, $28.7 \%$ gained 2 or more BMI units at the start of the second pregnancy compared with $15 \%$ in the normal weight women [13]. Globally, nearly $50 \%$ of women have weight gain during pregnancy above the NAM guidelines [14] leading to an increased risk for postpartum weight retention $[15,16]$. On the other hand, weight loss in obese pregnant women was associated with reduced perinatal risks but not with the rate of low birth weight or small-forgestational-age (SGA) neonates in obese women from class III [17]. In this study, we therefore wanted to evaluate the relation between GWG and relevant perinatal outcomes in the light of the NAM recommendations, especially for obese pregnant women (2009-2014) to prevent maternal obesity in the longer run. 
Devlieger et al.: Gestational Weight Gain Recommendations in Obese Pregnant Women

\section{Subjects and Methods}

\section{Subjects}

Since 1996, the Flemish Study Centre for Perinatal Epidemiology (SPE) has been routinely registering perinatal data from all deliveries in Flanders. Perinatal data from all maternity units are collated centrally. Data are subjected to an error detection program, checked for accuracy and completeness with extreme (considered outliers) or missing values confirmed with individual maternity units where appropriate [18]. Definitions used for stillbirths or live births of infants with a weight of $\geq 500 \mathrm{~g}$ are in agreement with those from the World Health Organization and the Fédération Internationale de Gynécologie et d'Obstétrique [19]. Data compiled included maternal and gestational age (completed weeks) at delivery, maternal height and weight before pregnancy and weight at delivery (since 2009), parity, hypertension in pregnancy, diabetes in pregnancy, mode of delivery, birth weight, congenital malformations and perinatal mortality.

We performed an epidemiological analysis of women delivering a singleton term $(\geq 37$ weeks) live birth between 2009 and 2014. We included all 371,117 term singleton deliveries from 2009 to 2014 (91.8\%). We excluded multiple pregnancies as these pregnancies have specific guidelines regarding GWG. We furthermore excluded 1,848 perinatal deaths because of missing values for BMI and GWG. Ranges of maternal height (1.35-1.95 m), prepregnancy weight (35-170 kg), maternal weight at delivery ( $40-185 \mathrm{~kg})$ and GWG $(-25$ to $+50 \mathrm{~kg})$ were found to be realistic ranges for inclusion in the analysis. After excluding missing values for all relevant variables of interest, a total of 337,590 (83.6\%) pregnancies were included in the analysis. For the multivariate analysis, we excluded patients developing preeclampsia and gestational diabetes, as fluid expansion and dietary interventions, respectively, may be a confounding factor for weight change $(n=9,628)$.

\section{Outcome Variables}

The BMI was categorized according to the World Health Organization [20] and the NAM [8] matched cutoffs, that is, for underweight women BMI $<18.5$, for normal weight 18.5-24.9, for overweight 25-29.9, for obese class I 30-34.9, for obese class II 35-39.9 and for obese class III $\geq 40$. Gestational weight change was calculated by subtracting the maternal prepregnancy weight from the weight at delivery and was categorized by pregestational BMI in accordance with the 2009 NAM guidelines for adequate GWG (for underweight women, adequate GWG was defined as between 12.5 and $18 \mathrm{~kg}$, for normal weight women between 11.5 and $16 \mathrm{~kg}$, for overweight between 7 and $11 \mathrm{~kg}$ and for the obese between 5 and $9 \mathrm{~kg}$ ). Prepregnancy weight and height were self-reported during pregnancy. Maternal weight at delivery was measured in the delivery room or, if not available, the weight at the last prenatal visit was used. In case of extreme values (considered outliers) or missing values, the maternity units were contacted for confirmation or correction.

We considered both maternal and neonatal outcomes. Maternal outcomes of interest included rates of gestational hypertension and emergency cesarean section. The diagnosis of gestational hypertension is defined according to the criteria of the International Society for the Study of Hypertension in Pregnancy as a systolic blood pressure reading $\geq 140 \mathrm{~mm} \mathrm{Hg}$ and/or a diastolic blood pressure $\geq 90 \mathrm{~mm} \mathrm{Hg}$ (average of at least 2 measurements taken at least 15 min apart) [21].

Neonatal parameters of interest included macrosomia $(\geq 4,000 \mathrm{~g})$, being large for gestational age (LGA, $>90$ th percentile), SGA $(<10$ th percentile), low birth weight $(<2,500 \mathrm{~g})$ and admission to the neonatal intensive care unit. SGA and LGA were defined using regional population-specific references $[2,13]$.

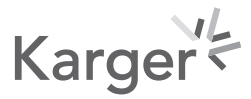


Devlieger et al.: Gestational Weight Gain Recommendations in Obese Pregnant Women

A

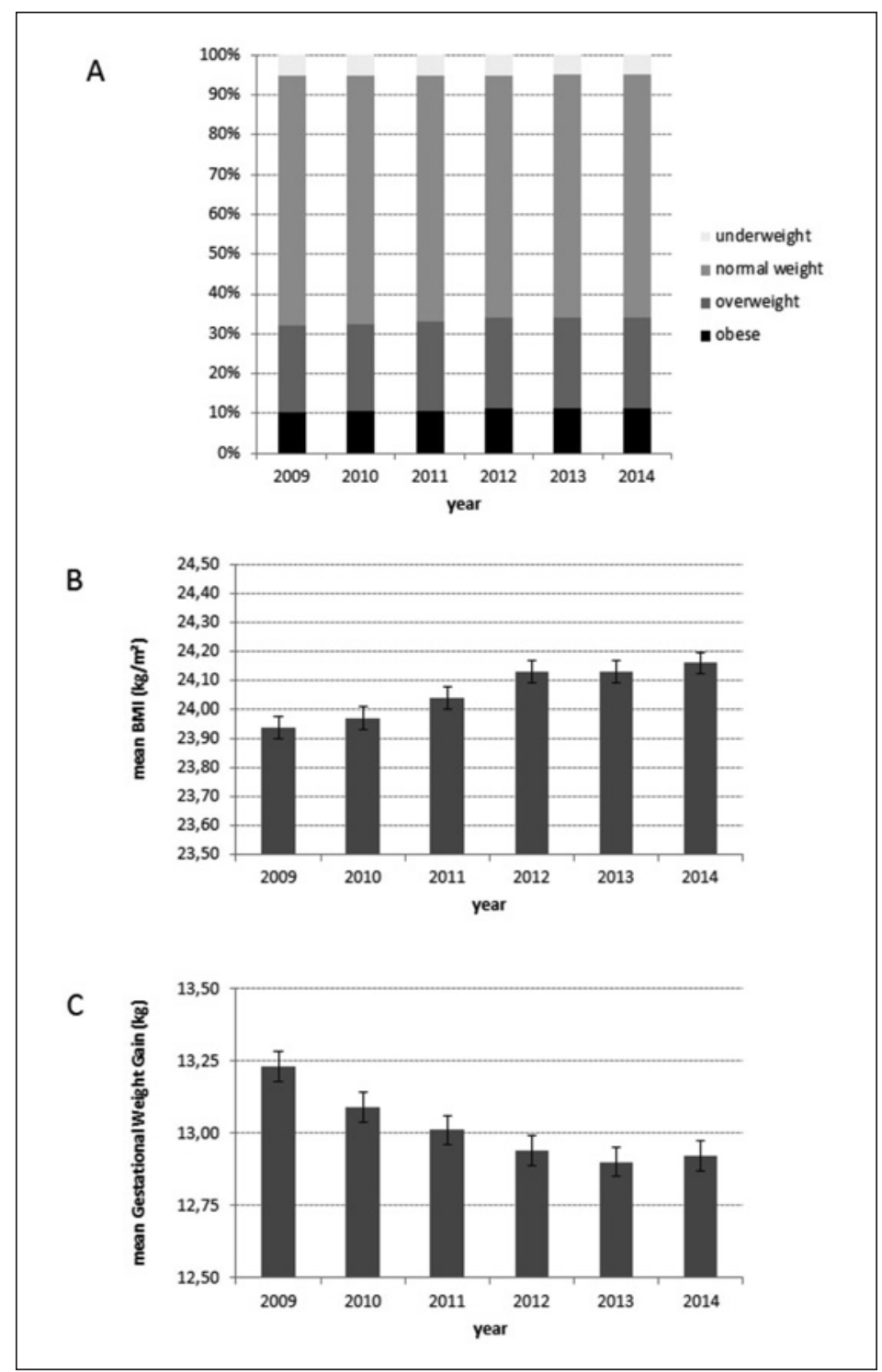

B

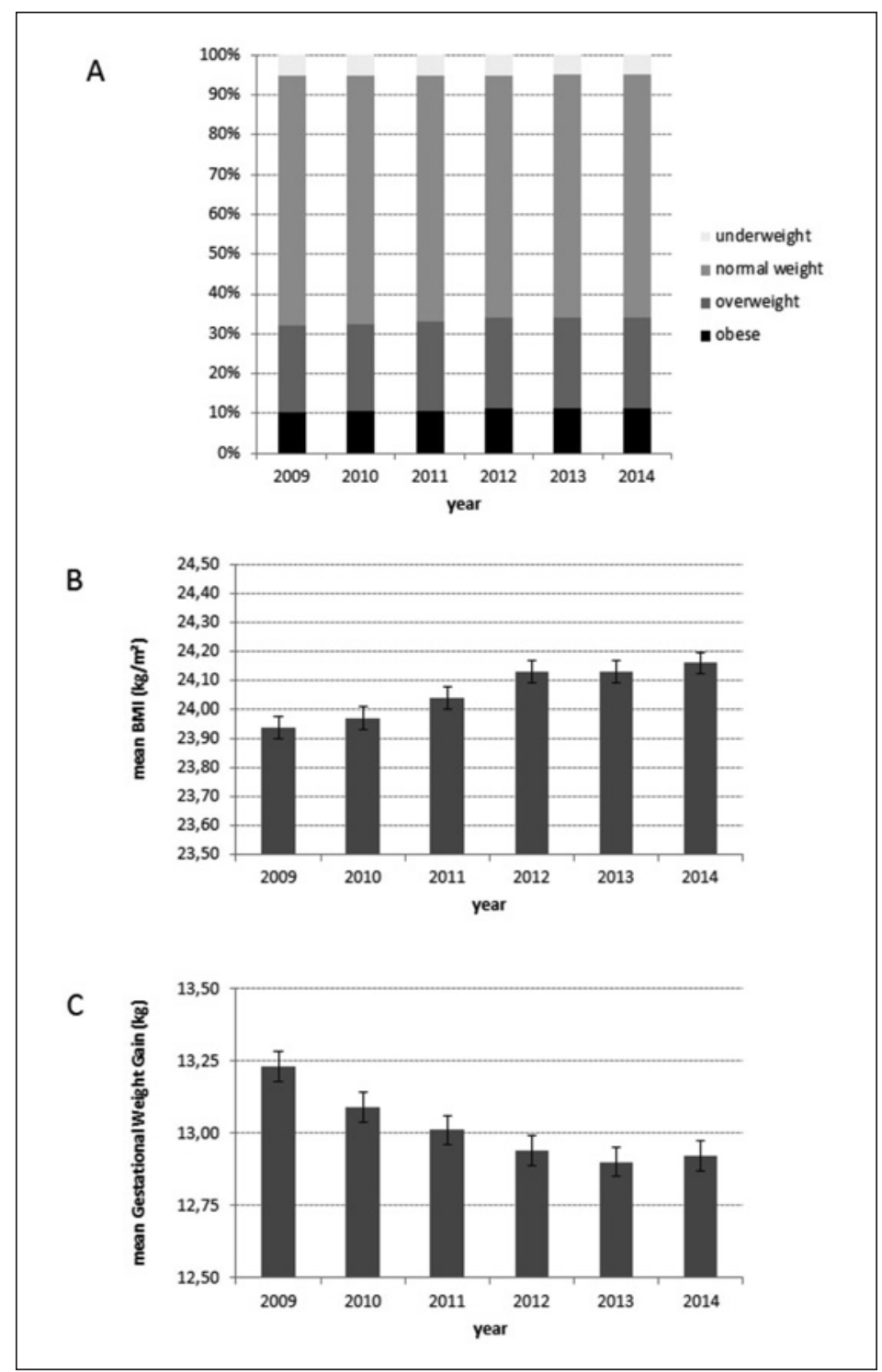

Fig. 1. Body mass index (BMI) and gestational weight gain (GWG) from 2009 to 2014. A Prevalence of maternal obesity. B Mean BMI. C Mean GWG.

\section{Statistical Methods}

In univariate analysis, to assess differences in categorical variables, the likelihood ratio $\chi^{2}$ and Mantel-Haenszel $\chi^{2}$ tests were used. To assess differences in continuous variables, the $t$ test was used. The Wald $95 \%$ confidence limits of a proportion were calculated.

For the multivariate analyses, we only considered 7 of the 8 outcomes as we excluded patients with gestational diabetes $(n=9,628)$. For each of the 7 outcomes, we built a logistic regression model to assess the impact of gestational weight change on the outcome adjusting for parity, maternal age, mode of conception (spontaneous, assisted), gestational age and year of delivery. The adjusted odds ratios (aOR) were reported. The logistic regression models were performed twice: once with gestational weight change as a categorical variable and once as a continuous variable. For multiparous women of maternal age of 30 years, spontaneous conception, gestational age of 39 weeks and delivery in 2014, we calculated the predicted probability on an adverse outcome for each possible value of gestational weight change. Those predicted probabilities have been visualized in a graph. The optimal GWG was considered as the point where the curves of SGA and LGA cross. All statistical analyses were performed with SAS 9.4. 
Devlieger et al.: Gestational Weight Gain Recommendations in Obese Pregnant Women

\section{Results}

BMI and GWG 2009-2014

The prevalence of maternal obesity increased from $10.3 \%$ in 2009 to $11.4 \%$ in 2014 ( $p<$ 0.001 ; Fig. $1 \mathrm{~A}$ ). Concurrently, the proportion of women starting pregnancy with a healthy BMI decreased from 62.9 to $61.1 \%(p<0.001)$.

Within the obese pregnant population $(n=36,791)$, most $(72 \%)$ were moderately obese (class I, BMI 30-34.9), 21\% were extremely obese (class II, BMI 35-39.9) and 7\% morbidly obese (class III, BMI $\geq 40$ ).

The mean BMI increased from 23.9 (4.5 SD) in 2009 to 24.2 (4.6 SD) in $2014(p<0.001$; Fig. 1B). Maternal age and parity both increased during the study period: mean maternal age from 29.6 years (4.8 SD) to 30.2 years ( $4.7 \mathrm{SD}$ ), and the proportion of parous women from $53.2 \%$ (confidence interval, CI, 52.7-53.6) to 56.3\% (CI 55.9-56.7). Obese pregnant women were older, were more often multiparous, and pregnancy more often resulted from assisted reproduction (Table 1).

Gaining weight within the 2009 NAM GWG recommendations was globally poor in the total population $(38.0 \%)$ and was the highest in the normal weight group (42.3\%) and lowest in the overweight (30.2\%) and obese groups (28.2\%).

The mean GWG in the total population from 2009 to 2014 decreased over the years and leveled off from 2012 onwards (Fig. 1C). Despite this, more than 1 in 2 overweight (57\%) and obese pregnant women (53\%) gained weight above the NAM recommendations.

Excessive GWG was most frequent in younger primiparous women and was associated with hypertensive complications, induction of labor, emergency cesarean delivery and LGA babies within all BMI categories (Table 1).

\section{Perinatal Outcomes 2009-2014}

Time Effects

In the univariate analysis, the prevalence of diabetes in pregnancy significantly increased between 2009 and 2014 from $2.1 \%$ (CI 2.0-2.3) to 3.5\% (CI 3.3-3.6; $p<0.001$; table not shown). In the multivariate analysis, no significant changes in the prevalence of hypertension in pregnancy, macrosomia $(\geq 4,000 \mathrm{~g})$, low birth weight $(<2,500 \mathrm{~g})$ and SGA babies were observed over the last 6 years within the different BMI categories. However, in class III obese women, the aOR for LGA babies have been decreasing while in overweight women, the aOR for emergency cesarean and neonatal intensive care unit admission have increased (table not shown).

\section{BMI and GWG Effects}

Excessive GWG was associated with an increased risk for pregnancy-induced hypertension. This additive effect was more pronounced in the higher BMI categories. For example, hypertensive complications were present in $4.4 \%$ of the patients showing excessive GWG and having a normal BMI, versus $22.3 \%$ in class III obese women with excessive GWG (Table 2).

Odds ratios for macrosomia and LGA were also significantly higher if women gained weight beyond compared to within recommendations, with increasing prevalence in higher BMI classes. For example, the estimated probability of delivering an LGA baby was $16 \%$ if a normal weight woman (multiparous, age 30, gestational age at delivery 39 weeks, spontaneous conception, delivery in 2014) gained more than the recommended weight compared to $19 \%$ in a class I obese woman gaining more than the recommended weight, and $23 \%$ in class II or III obese women.

Class III obese women did not show a significant increase in risk for macrosomia if they gained more than the recommended weight (aOR 1.22, 95\% CI 0.94-1.59; $p=0.14$ ), but the risk for LGA babies (aOR 1.29, 95\% CI 1.01-1.65, $p=0.04$ ) increased when exceeding the 
Devlieger et al.: Gestational Weight Gain Recommendations in Obese Pregnan Women

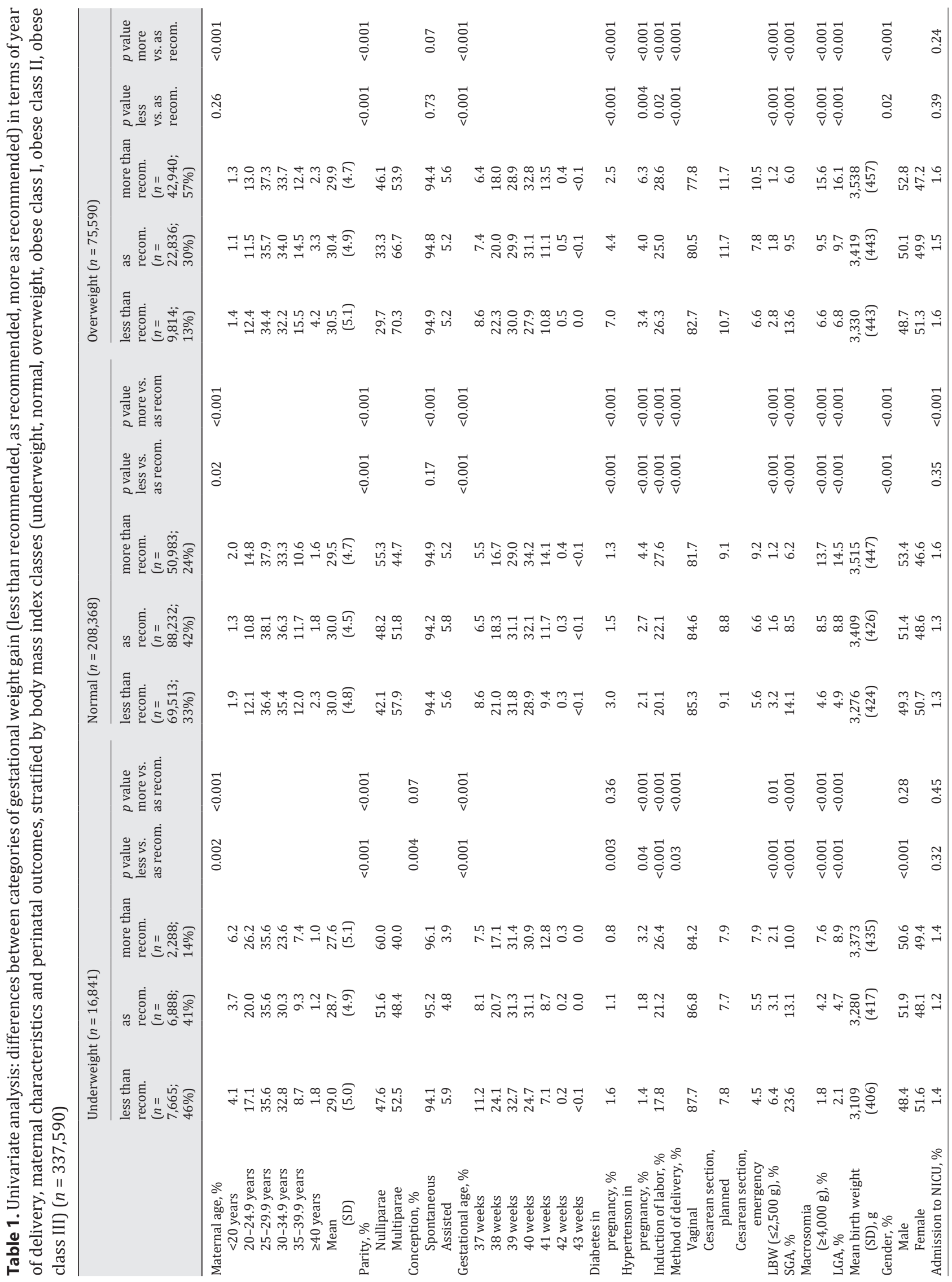


Devlieger et al.: Gestational Weight Gain Recommendations in Obese Pregnant Women

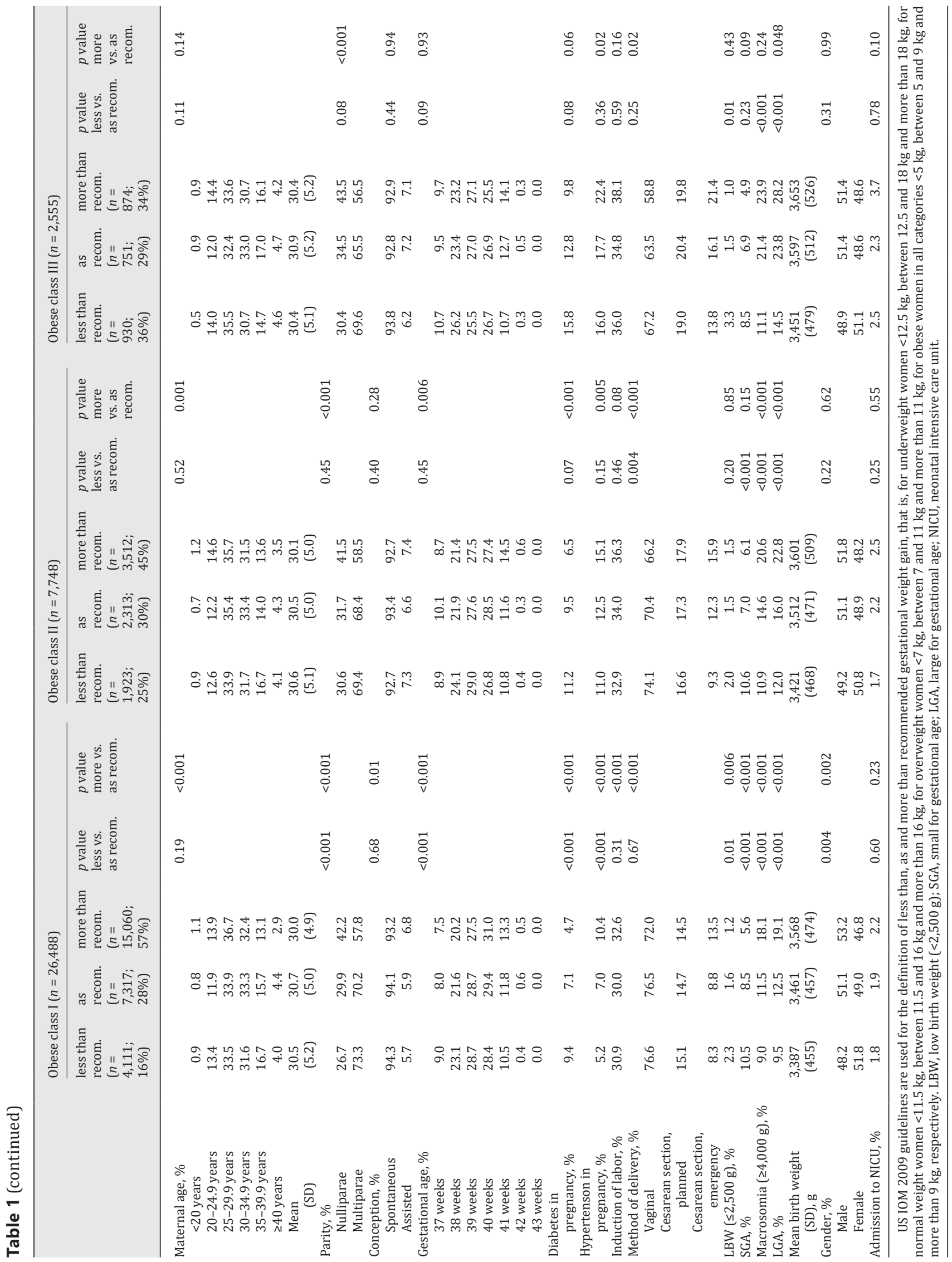


Devlieger et al.: Gestational Weight Gain Recommendations in Obese Pregnant

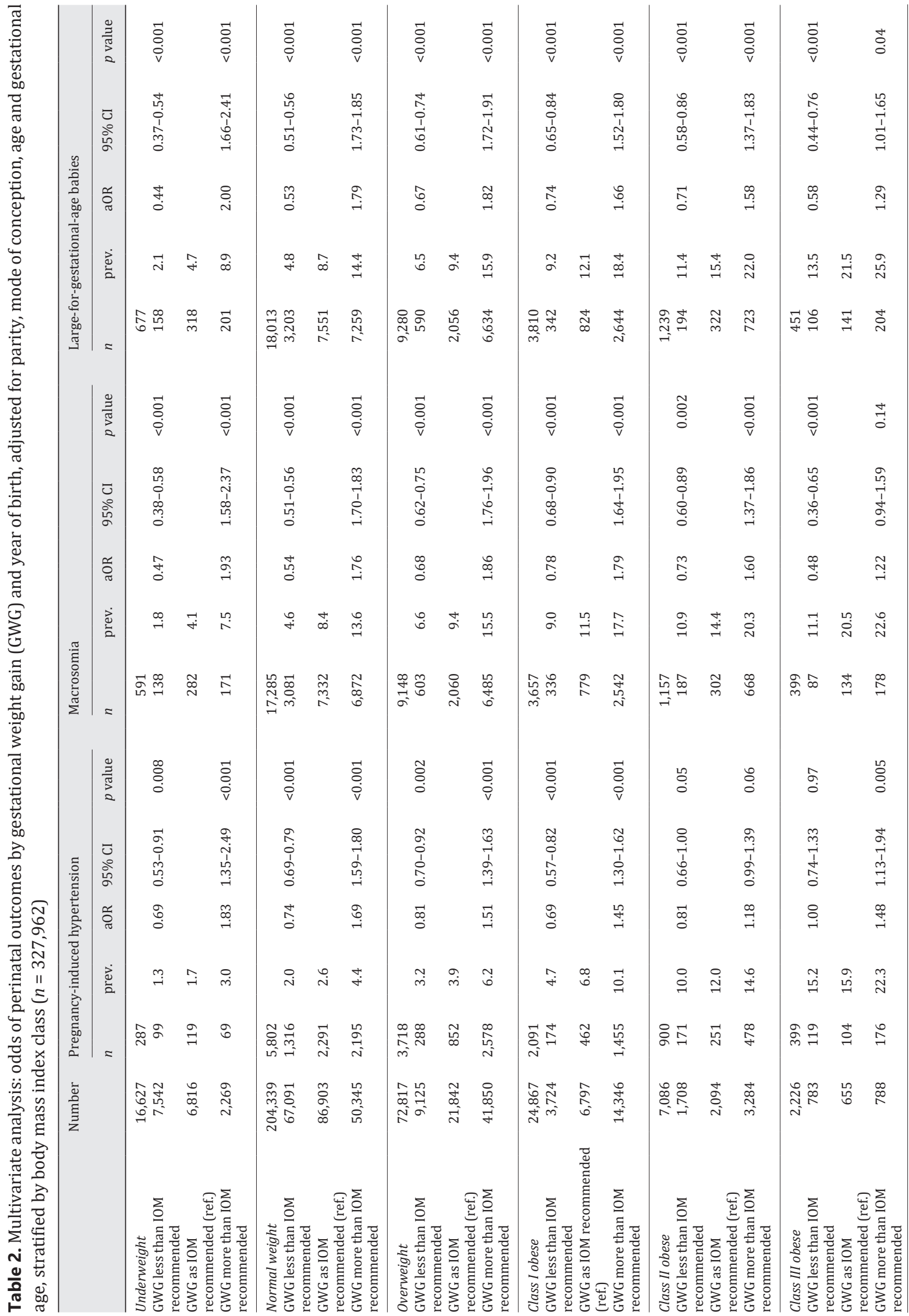




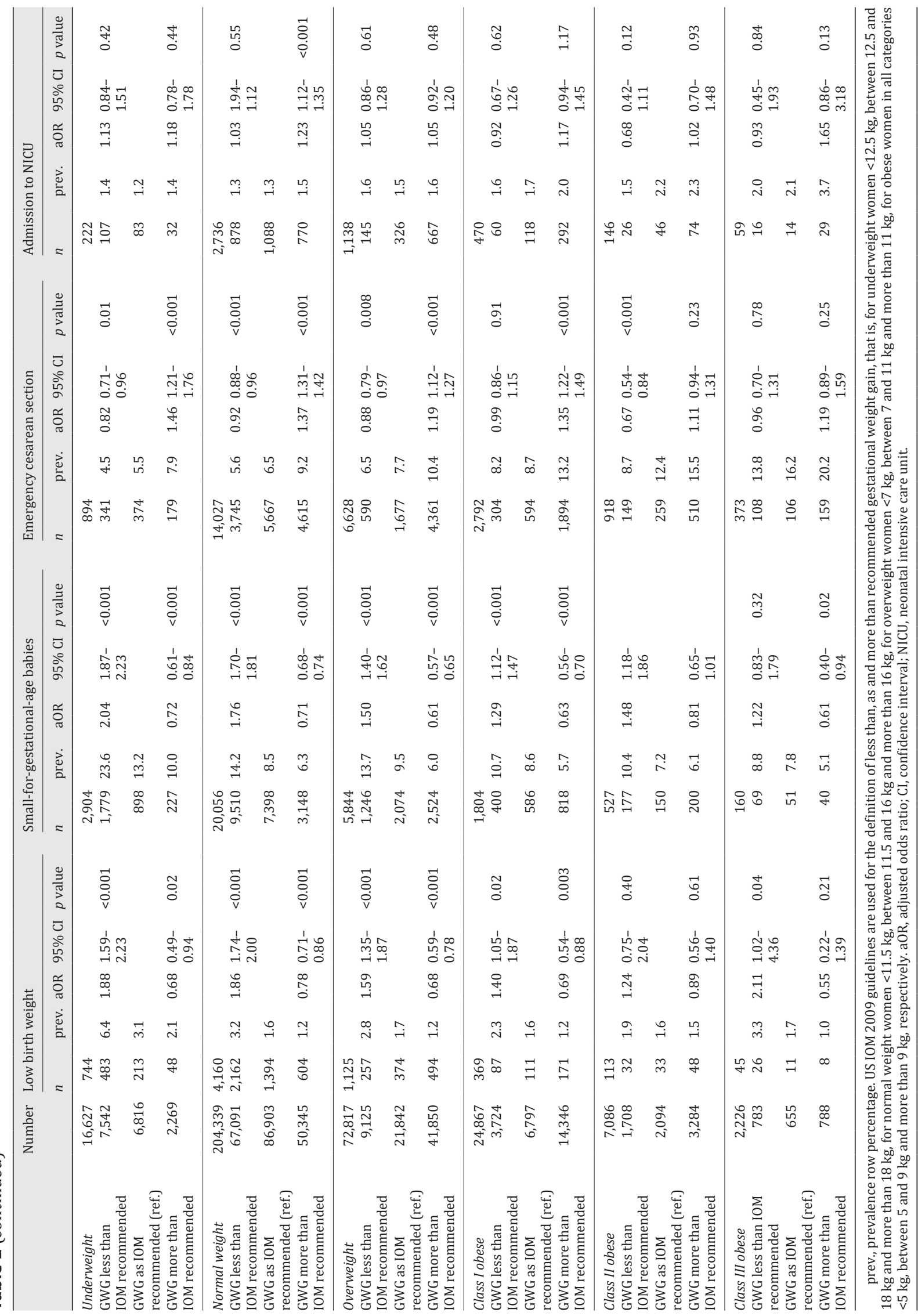


Devlieger et al.: Gestational Weight Gain Recommendations in Obese Pregnant

A

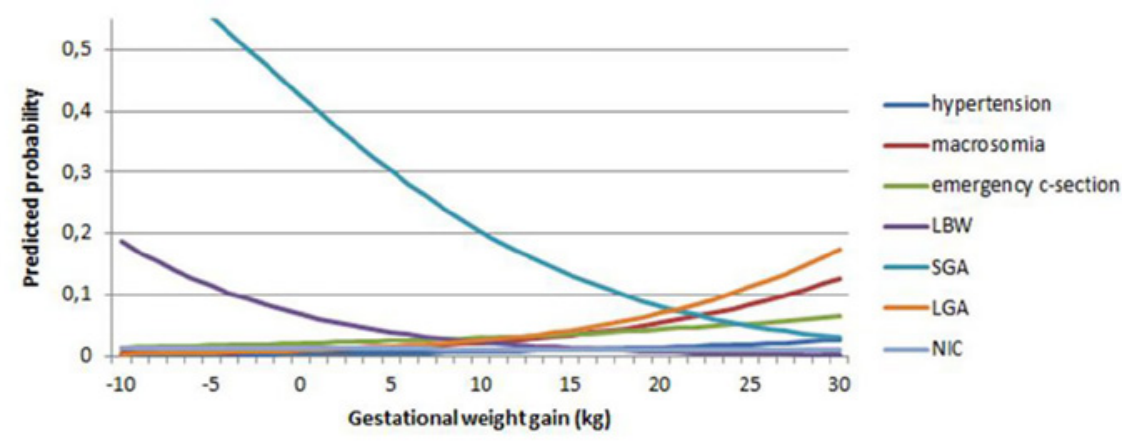

B

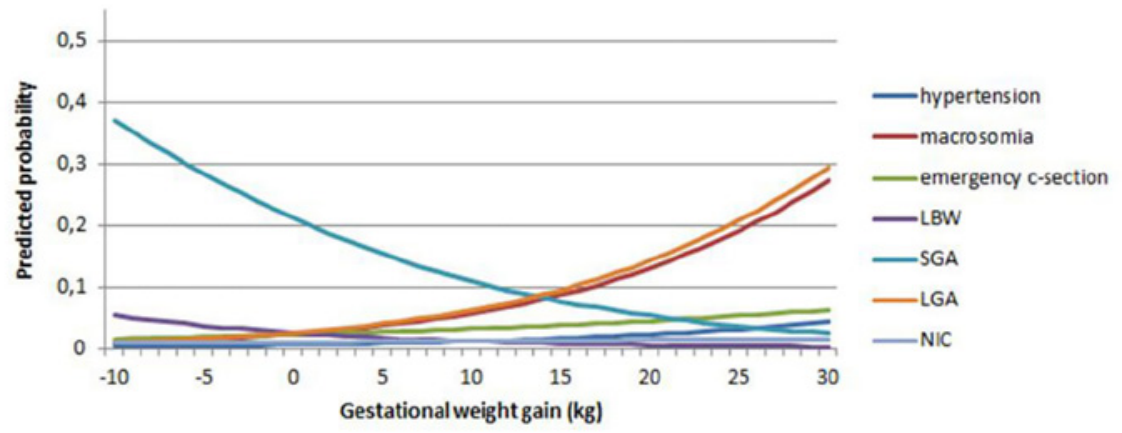

C

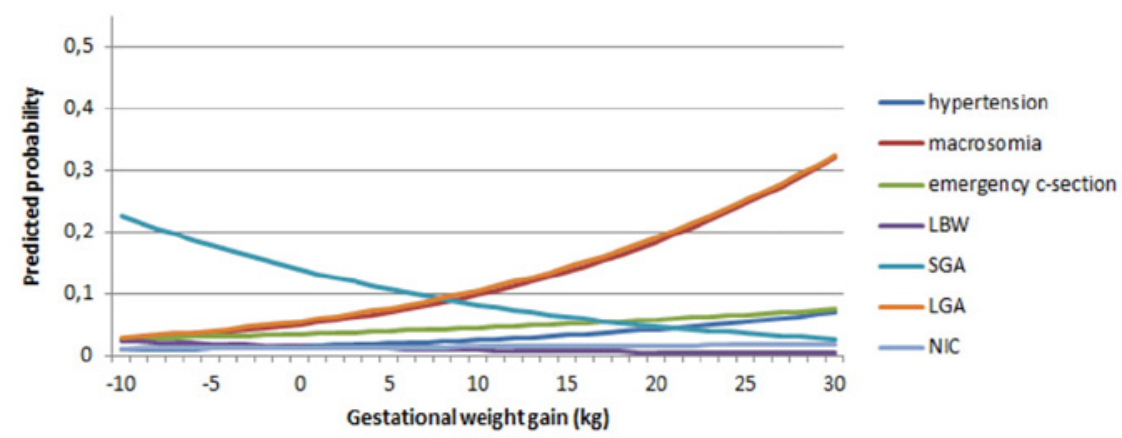

Fig. 2. A-F Different predicted probabilities for each BMI category and for the different classes of obesity. c-section, cesarean section; LBW, low birth weight; SGA, small for gestational age; LGA, large for gestational age; NIC, neonatal intensive care.

(Figure continued on next page.)

guidelines. GWG did not influence the risk for delivering SGA babies in obese class III women (8.8 vs. $7.8 \%$, aOR $1.22,95 \%$ CI $0.83-1.79, p=0.32$ for women gaining weight within vs. beyond recommendations).

The aOR for emergency cesarean section increased with increasing GWG in underweight, normal weight and overweight women. For obese women of class II and III however, this trend was not identifiable (Table 2).

\section{Prediction Model}

In the multivariate analysis using GWG as a continuous variable, we calculated the predicted probability for relevant adverse outcomes for each possible value of GWG. We made a model using a 30-year-old multiparous woman in 2014, delivering at 39 weeks after 
Devlieger et al.: Gestational Weight Gain Recommendations in Obese Pregnant

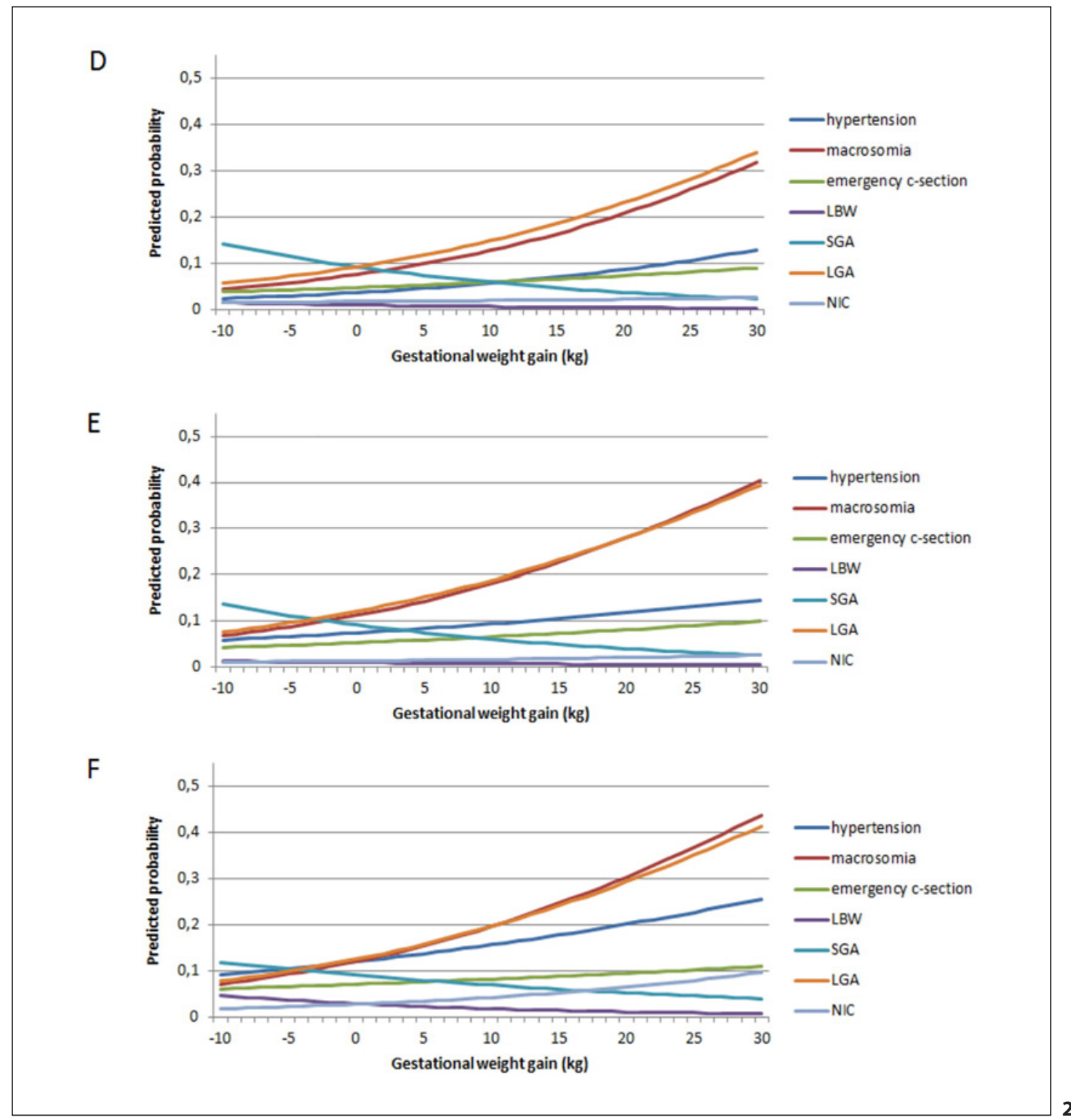

a spontaneous conception. The different predicted probabilities have been visualized for each BMI category, and for the different classes of obesity (Fig. 2A-F). LGA and SGA were the most frequent outcomes. The lowest predicted probabilities of LGA and SGA infants were associated with a weight gain of $21 \mathrm{~kg}$ in underweight women, $14 \mathrm{~kg}$ in normal weight women and about $8 \mathrm{~kg}$ in overweight women. In obese women, the lowest probability coincided with $0 \mathrm{~kg}$ weight gain in class I, $4 \mathrm{~kg}$ weight loss in class II, and $5 \mathrm{~kg}$ weight loss in class III.

\section{Discussion}

This study reports on a unique high-quality data set of over 335,000 pregnant women, focusing on the evolution of BMI and GWG and their impact on relevant perinatal outcomes. We show that the problem of maternal obesity remains endemic in our region. One in 3 women was overweight or obese at the start of pregnancy, and the prevalence of class II and 
Devlieger et al.: Gestational Weight Gain Recommendations in Obese Pregnant Women

III obesity increased during the study period. Moreover, our study results challenge the current NAM recommendations for GWG in obese women, especially in those with class II and III obesity. Obesity in women of reproductive age adversely affects current and future generations, with implications from preconception to pregnancy and the postpartum period [22]. It therefore requires more attention from the public, caregivers and funders.

\section{Maternal BMI and Pregnancy Outcome}

Our data confirm the association of maternal obesity with adverse maternal, fetal and neonatal outcomes reported in other studies [17, 22, 23]. The association between hypertensive complications and emergency cesarean section was striking. In normal weight women with adequate GWG, the risk for hypertensive complication or undergoing emergency cesarean section was 1.8 and $6.6 \%$, respectively, compared to 17.7 and $16.1 \%$ in morbidly obese women. This is worrying, especially as urgent abdominal deliveries in (morbidly) obese patients are far more costly, technically challenging and associated with morbidity [24]. Besides, this is an increasing risk group for inadequate gestational age including extreme preterm and post-term births [25]. Our results may suggest that GWG largely determines adverse perinatal outcomes. However, when comparing plots for the different BMI categories (Fig. 2), it becomes clear that the effect of GWG is superimposed on the stronger determinant being the prepregnancy BMI. This has also been demonstrated in other studies from our group and others [2, 14, 26-28]. A recent meta-analysis reporting on a $264 \%$ increased odds of child obesity when mothers have prepregnancy obesity, provides substantial evidence for the need to develop early interventions prior to conception, to support women of childbearing age with weight management in order to tackle intergenerational obesity [29].

\section{Additive Effect of GWG}

As expected, excessive GWG had an additive effect on the deleterious effect of starting the pregnancy with a high BMI. The risk for an LGA newborn in normal weight till class II obese women increased from 8.7 to $14.4 \%$ and from 15.4 to $22.0 \%$, respectively, depending on whether women gained weight within or above recommendations. This additive effect was less predictable in class III obese women (21.5-25.9\%). Yet, GWG in obese women [30] as well as in normal weight women [31] is a modifiable factor during pregnancy, and therefore recommendations are important to inform the patients and direct caregivers [32]. Diet and physical activity-based interventions are most effective in reducing GWG, with only a small impact on reducing cesarean delivery (OR $0.91,95 \%$ CI $0.83-0.99$ ) [33]. Unfortunately, a recent study showed that obese pregnant women should be counseled on healthy eating, physical activity and sedentary behavior to reduce neonatal adiposity in their offspring [34].

\section{Modeling "Optimal" GWG}

Using GWG as a continuous variable, we calculated windows of GWG associated with "optimal" pregnancy outcome for the different BMI categories, including the different classes of obesity. The "optimal" pregnancy outcome was defined as the GWG that was associated with the lowest incidence of pregnancy complications related to both maternal BMI and GWG, especially LGA and SGA. These are the most frequent adverse outcomes registered in the database, and both are associated with adverse long-term metabolic outcome for the children. Being born LGA has been shown to be a stronger marker for risk of being overweight/obese in early childhood compared with maternal diabetes during pregnancy [35]. SGA neonates on the other hand have a significantly increased risk for cardiovascular dysfunction in early childhood [36].

The calculated "most optimal" GWG corresponds well with the current NAM guidelines for the underweight, normal weight and overweight categories and therefore supports the

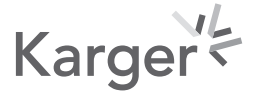


Devlieger et al.: Gestational Weight Gain Recommendations in Obese Pregnant Women

validity and use of these guidelines in our population. In contrast, the modeling results obtained in the different classes of obesity were substantially different. In women with class III obesity, the NAM recommends a total weight gain between 5 and $9 \mathrm{~kg}$ during pregnancy. Our data suggest that patients in this class only have a low probability of weight-related complications if they lose weight during pregnancy, which was also confirmed by others [37]. For class I and II obesity women, the calculated optimal GWG windows were also lower than the advised 5-9 kg advised by the NAM, irrespective of the degree of obesity.

\section{Lessons for Clinical Practice}

The safety of weight loss in obese mothers during pregnancy is increasingly being reported in epidemiological studies $[17,38]$ but needs to be confirmed in prospective studies. Our data suggest that the current guidelines are insufficiently stringent for the obese categories and that differentiation between the different classes of obesity would be a helpful addition to the current guidelines. The need for more refined GWG guidelines or tailored to a specific national situation on GWG has been recently reported based on an analysis of the Swedish maternal health care register [39]. A recent systematic review on perinatal outcomes related to GWG categories per BMI group showed that GWG below recommendations was associated with a higher risk for SGA (OR 1.53) and preterm birth (OR 1.70) [14]. However, although they distinguished between classes of obesity as well as between weight loss and GWG below guidelines, they could not report on the lowest prevalence for SGA and LGA based on GWG as a continuous variable as we did in our analysis.

Other researchers have recently recognized that the NAM guidelines are probably too liberal and have developed interventions aiming at limiting GWG in obese pregnant women to a maximum of $5 \mathrm{~kg}$ or less [40]. In the DALI study, this approach led to a significant reduction in GWG, an improved glucose metabolism and neonatal adiposity [34, 41]. The importance of limiting GWG has led to the development and testing of numerous lifestyle interventions. However, disappointingly, the majority have resulted in an insufficient reduction of GWG to have a meaningful impact on pregnancy and birth outcomes. Concurrently, the impact of these lifestyle interventions on rates of obesity in the offspring remains to be determined [7, 42, 43].

The most effective prevention is encouraging women to embark on pregnancy with a healthy lifestyle and BMI. Preconception lifestyle programs are seldom offered to young adult women presenting for contraception or routine health care visits [44]. Furthermore, the content and effectiveness of those programs currently remain unproven [42]. Postpartum weight retention also negatively influences the prepregnancy BMI in a subsequent pregnancy. Recently, more attention has been given to the problem of weight retention after pregnancy, its impact on subsequent pregnancies $[13,45,46]$ and on the life-long health of the women [7]. Research has focused on possible interventions in the so-called "interpregnancy period," the period between 2 pregnancies. Ideally, interpregnancy interventions should not only focus on the postpartum period, but also the subsequent pregnancy. Currently, several populations are under evaluation, including patients in a large multicenter randomized trial in the region studied in this paper [47] (www.interactstudy.org).

\section{Strengths and Limitations}

The strength of this analysis resides in the amount of well-documented pregnancies and the quality of the registration in the region of Flanders. Indeed, this register covers almost all deliveries in the region including most home deliveries. Furthermore, the scrutiny with which the missing data are traced contributes to the quality of this internationally recognized register.

Sociodemographic variables like ethnicity were not included in this analysis but were evaluated and reported in this Caucasian North-European population elsewhere in detail for

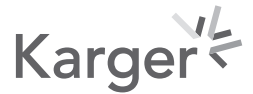


Devlieger et al.: Gestational Weight Gain Recommendations in Obese Pregnant Women

the year 2009, coinciding with the start period of this study [2]. Theoretically, sociodemographic changes, especially age, parity and ethnic background could potentially influence BMI, GWG and their respective influence on the perinatal outcomes. The study window is however relatively small, and these factors have been very stable over that study period [18]. It is therefore very improbable that the effect of sociodemographic changes results in the different findings or conclusions in this paper. We need to acknowledge the possible underestimation of self-reported prepregnancy maternal weight as this may affect the calculation of prepregnancy BMI and subsequently influence the calculation of GWG. The link between prepregnancy BMI, GWG and measured correlates may be affected by this potential error. A possible difference in maternal weight between the last prenatal visit and maternal weight at delivery is not significantly meaningful in this large term cohort of more than 330,000 women.

Besides reporting on the optimal weight gain by using the lowest intersection between LGA and SGA, one has to take also into account the immediate and longer-term consequences of excessive GWG for the mothers, especially in those from class II and III obesity. Although a rather low incidence of gestational diabetes mellitus (GDM) which is related to different screening policies, that is, Diabetic Pregnancy Study Group versus Carpenter-Coustan cutoffs, GDM is an important complication of obesity with long-term impact on the health of mothers and offspring [6]. GDM increased substantially in this population over the study period. Omission of GDM due to inconsistent primary data (influence of diet treatment) is a weakness of all studies addressing optimal GWG [14]. Measurement of GWG up to the 2nd trimester could give important information on this important outcome. Unfortunately, these data are seldom available. And lastly, findings from this analysis cannot be interpreted as causalities but rather as correlational, because of the cross-sectional design.

In summary, this paper provides a novel insight into the increasing trend in maternal obesity and associated outcomes, and challenges the current recommendations for GWG in obese women, especially in those from classes II and III.

\section{Acknowledgment}

The authors want to thank Patrice Eastwood, MD, for linguistic advice.

\section{Statement of Ethics}

The scientific committee of the SPE granted approval for the analysis of the anonymized data. This study was exempt from approval by an Institutional Review Board because data were used for scientific purposes only.

\section{Conflict of Interest Statement}

The authors have no conflicts of interest to declare. All authors have completed the ICMJE uniform disclosure form at http://www.icmje.org/coi_disclosure.pdf and declare: no support from any organization for the submitted work; no financial relationships with any organizations that might have an interest in the submitted work in the previous 3 years, and no other relationships or activities that could appear to have influenced the submitted work.

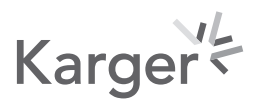


Devlieger et al.: Gestational Weight Gain Recommendations in Obese Pregnant Women

\section{Funding Sources}

R.D. is a fundamental clinical investigator for the Flemish Research Fund (FWO Fundamental Clinical Investigatorship 1803311N). This paper was realized using data from the SPE, supported by the Flemish agency for Care and Health (VAZG).

\section{Author Contributions}

R.D. and A.B. designed the study protocol, together with L.A. who also coordinated and executed the statistical analysis. R.G. supported in the data cleaning, T.N. coordinated the literature searches and revised the final script.

\section{References}

1 Flegal KM, Kruszon-Moran D, Carroll MD, Fryar CD, Ogden CL. Trends in Obesity Among Adults in the United States, 2005 to 2014. JAMA. 2016 Jun;315(21):2284-91.

2 Bogaerts A, Van den Bergh B, Nuyts E, Martens E, Witters I, Devlieger R. Socio-demographic and obstetrical correlates of pre-pregnancy body mass index and gestational weight gain. Clin Obes. 2012 Oct;2(5-6):150-9.

3 Devlieger R, Benhalima K, Damm P, Van Assche A, Mathieu C, Mahmood T, et al. Maternal obesity in Europe: where do we stand and how to move forward?: A scientific paper commissioned by the European Board and College of Obstetrics and Gynaecology (EBCOG). Eur J Obstet Gynecol Reprod Biol. 2016 Jun;201:203-8.

4 Kirkegaard H, Stovring H, Rasmussen KM, Abrams B, Sørensen TI, Nohr EA. How do pregnancy-related weight changes and breastfeeding relate to maternal weight and BMI-adjusted waist circumference $7 \mathrm{y}$ after delivery? Results from a path analysis. Am J Clin Nutr. 2014 Feb;99(2):312-9.

5 Gaillard R, Welten M, Oddy WH, Beilin LJ, Mori TA, Jaddoe VW, et al. Associations of maternal prepregnancy body mass index and gestational weight gain with cardio-metabolic risk factors in adolescent offspring: a prospective cohort study. BJOG. 2016 Jan;123(2):207-16.

6 Catalano PM, Shankar K. Obesity and pregnancy: mechanisms of short term and long term adverse consequences for mother and child. BMJ. 2017 Feb;356:j1.

7 Godfrey KM, Reynolds RM, Prescott SL, Nyirenda M, Jaddoe VW, Eriksson JG, et al. Influence of maternal obesity on the long-term health of offspring. Lancet Diabetes Endocrinol. 2017 Jan;5(1):53-64.

8 Rasmussen KM, Yaktine AL (eds). Weight Gain During Pregnancy: Reexamining the Guidelines. Institute of Medicine; National Research Council 2009:696.

9 Heslehurst N, Ells LJ, Simpson H, Batterham A, Wilkinson J, Summerbell CD. Trends in maternal obesity incidence rates, demographic predictors, and health inequalities in 36,821 women over a 15-year period. BJOG. 2007 Feb;114(2):187-94.

10 Ogden CL, Carroll MD, Kit BK, Flegal KM. Prevalence of childhood and adult obesity in the United States, 20112012. JAMA. 2014 Feb;311(8):806-14.

11 Ogden CL, Carroll MD, Lawman HG, Fryar CD, Kruszon-Moran D, Kit BK, et al. Trends in Obesity Prevalence Among Children and Adolescents in the United States, 1988-1994 Through 2013-2014. JAMA. 2016 Jun; 315(21):2292-9.

12 Drieskens S. Gezondheidsenquête 2013. Rapport 2: Gezondheidsgedrag en leefstijl. WIV-ISP, Brussel; 2014.

13 Bogaerts A, Van den Bergh BR, Ameye L, Witters I, Martens E, Timmerman D, et al. Interpregnancy weight change and risk for adverse perinatal outcome. Obstet Gynecol. 2013 Nov;122(5):999-1009.

14 Goldstein RF, Abell SK, Ranasinha S, Misso M, Boyle JA, Black MH, et al. Association of Gestational Weight Gain With Maternal and Infant Outcomes: A Systematic Review and Meta-analysis. JAMA. 2017 Jun;317(21):2207-25.

15 Bogaerts AF, Van den Bergh BR, Witters I, Devlieger R. Anxiety during early pregnancy predicts postpartum weight retention in obese mothers. Obesity (Silver Spring). 2013 Sep;21(9):1942-9.

16 Bogaerts A, De Baetselier E, Ameye L, Dilles T, Van Rompaey B, Devlieger R. Postpartum weight trajectories in overweight and lean women. Midwifery. 2017 Jun;49:134-141

17 Bogaerts A, Ameye L, Martens E, Devlieger R. Weight loss in obese pregnant women and risk for adverse perinatal outcomes. Obstet Gynecol. 2015 Mar;125(3):566-75.

18 Devlieger R, Martens E, Martens G, Van Mol C, Cammu H. Perinatale Activiteiten in Vlaanderen, 2014. Brussel: Vzw Studiecentrum voor Perinatale Epidemiologie. SPE; 2015.

19 Dunn PM. History of the FIGO committee on standardization of annual reports and definitions of medical terms 1964-1994. Int J Gynaecol Obstet. 2007 Feb;96(2):138.

20 World Health Organisation. Obesity and overweight. Fact sheet $N^{\circ} 311.2015$; updated January 2015.

21 Butalia S, Audibert F, Côté AM, Firoz T, Logan AG, Magee LA, et al.; Hypertension Canada. Hypertension Canada's 2018 Guidelines for the Management of Hypertension in Pregnancy. Can J Cardiol. 2018 May;34(5):526-31. 
Devlieger et al.: Gestational Weight Gain Recommendations in Obese Pregnant Women

22 Poston L, Caleyachetty R, Cnattingius S, Corvalán C, Uauy R, Herring S, et al. Preconceptional and maternal obesity: epidemiology and health consequences. Lancet Diabetes Endocrinol. 2016 Dec;4(12):1025-36.

23 Liu P, Xu L, Wang Y, Zhang Y, Du Y, Sun Y, et al. Association between perinatal outcomes and maternal prepregnancy body mass index. Obes Rev. 2016 Nov;17(11):1091-102.

24 Denison FC, Norwood P, Bhattacharya S, Duffy A, Mahmood T, Morris C, et al. Association between maternal body mass index during pregnancy, short-term morbidity, and increased health service costs: a populationbased study. BJOG. 2014 Jan;121(1):72-81.

25 Slack E, Best KE, Rankin J, Heslehurst N. Maternal obesity classes, preterm and post-term birth: a retrospective analysis of 479,864 births in England. BMC Pregnancy Childbirth. 2019 Nov;19(1):434.

26 Guelinckx I, Devlieger R, Beckers K, Vansant G. Maternal obesity: pregnancy complications, gestational weight gain and nutrition. Obes Rev. 2008 Mar;9(2):140-50.

27 Nohr EA, Vaeth M, Baker JL, Sørensen TI, Olsen J, Rasmussen KM. Combined associations of prepregnancy body mass index and gestational weight gain with the outcome of pregnancy. Am J Clin Nutr. 2008 Jun;87(6):1750-9.

28 Voerman E, Santos S, Inskip H, Amiano P, Barros H, Charles MA, et al.; LifeCycle Project-Maternal Obesity and Childhood Outcomes Study Group. Association of Gestational Weight Gain With Adverse Maternal and Infant Outcomes. JAMA. 2019 May;321(17):1702-15.

29 Heslehurst N, Vieira R, Akhter Z, Bailey H, Slack E, Ngongalah L, et al. The association between maternal body mass index and child obesity: A systematic review and meta-analysis. PLoS Med. 2019 Jun;16(6):e1002817.

30 Bogaerts AF, Devlieger R, Nuyts E, Witters I, Gyselaers W, Van den Bergh BR. Effects of lifestyle intervention in obese pregnant women on gestational weight gain and mental health: a randomized controlled trial. Int J Obes. 2013 Jun;37(6):814-21.

31 Thangaratinam S, Rogozinska E, Jolly K, Glinkowski S, Roseboom T, Tomlinson JW, et al. Effects of interventions in pregnancy on maternal weight and obstetric outcomes: meta-analysis of randomised evidence. BMJ. 2012 May;344(may16 4):e2088.

32 Dodd J, Thangaratinam S; i-WIP collaborative network. Researchers' position statement on tackling obesity in pregnancy: the International Weight Management in Pregnancy (i-WIP) collaboration pleads for public health intervention. BJOG. 2016 Jan;123(2):163-4.

33 Rogozińska E, Marlin N, Jackson L, Rayanagoudar G, Ruifrok AE, Dodds J, et al. Effects of antenatal diet and physical activity on maternal and fetal outcomes: individual patient data meta-analysis and health economic evaluation. Health Technol Assess. 2017 Aug;21(41):1-158.

34 van Poppel MN, Simmons D, Devlieger R, van Assche FA, Jans G, Galjaard S, et al. A reduction in sedentary behaviour in obese women during pregnancy reduces neonatal adiposity: the DALI randomised controlled trial. Diabetologia. 2019 Jun;62(6):915-25.

35 Kaul P, Bowker SL, Savu A, Yeung RO, Donovan LE, Ryan EA. Association between maternal diabetes, being large for gestational age and breast-feeding on being overweight or obese in childhood. Diabetologia. 2019 Feb;62(2):249-58.

36 Castagno M, Menegon V, Monzani A, Zanetta S, Secco GG, Rosso R, et al. Small-for-gestational-age birth is linked to cardiovascular dysfunction in early childhood. Am Heart J. 2019 Nov;217:84-93.

37 Kiel DW, Dodson EA, Artal R, Boehmer TK, Leet TL. Gestational weight gain and pregnancy outcomes in obese women: how much is enough? Obstet Gynecol. 2007 Oct;110(4):752-8.

38 Kominiarek MA, Seligman NS, Dolin C, Gao W, Berghella V, Hoffman M, et al. Gestational weight gain and obesity: is 20 pounds too much? Am J Obstet Gynecol. 2013 Sep;209(3):214.e1-11.

39 Nilses C, Persson M, Lindkvist M, Petersson K, Mogren I. High weight gain during pregnancy increases the risk for emergency caesarean section - Population-based data from the Swedish Maternal Health Care Register 2011-2012. Sex Reprod Healthc. 2017 Mar;11:47-52.

40 Poston L, Bell R, Croker H, Flynn AC, Godfrey KM, Goff L, et al.; UPBEAT Trial Consortium. Effect of a behavioural intervention in obese pregnant women (the UPBEAT study): a multicentre, randomised controlled trial. Lancet Diabetes Endocrinol. 2015 Oct;3(10):767-77.

41 Simmons D, Jelsma JG, Galjaard S, Devlieger R, van Assche A, Jans G, et al. Results From a European Multicenter Randomized Trial of Physical Activity and/or Healthy Eating to Reduce the Risk of Gestational Diabetes Mellitus: The DALI Lifestyle Pilot. Diabetes Care. 2015 Sep;38(9):1650-6.

42 Hanson M, Barker M, Dodd JM, Kumanyika S, Norris S, Steegers E, et al. Interventions to prevent maternal obesity before conception, during pregnancy, and post partum. Lancet Diabetes Endocrinol. 2017 Jan;5(1):65-76.

43 Dodd JM, Grivell RM, Louise J, Deussen AR, Giles L, Mol BW, et al. The effects of dietary and lifestyle interventions among pregnant women who are overweight or obese on longer-term maternal and early childhood outcomes: protocol for an individual participant data (IPD) meta-analysis. Syst Rev. 2017 Mar;6(1):51.

44 Ma RC, Schmidt MI, Tam WH, McIntyre HD, Catalano PM. Clinical management of pregnancy in the obese mother: before conception, during pregnancy, and post partum. Lancet Diabetes Endocrinol. 2016 Dec;4(12): 1037-49.

45 Villamor E, Cnattingius S. Interpregnancy weight change and risk of adverse pregnancy outcomes: a population-based study. Lancet. 2006 Sep;368(9542):1164-70.

46 McBain RD, Dekker GA, Clifton VL, Mol BW, Grzeskowiak LE. Impact of inter-pregnancy BMI change on perinatal outcomes: a retrospective cohort study. Eur J Obstet Gynecol Reprod Biol. 2016 Oct;205:98-104.

47 Bogaerts A, Ameye L, Devlieger R. Inter-pregnAncy Coaching for a Healthy fuTure (inter-act). March 15, 2017 ed: Clinicaltrials.gov; 2017. 